\title{
WIRELESS MAGNETIC SENSOR NETWORK FOR ROAD TRAFFIC MONITORING AND VEHICLE CLASSIFICATION
}

\author{
Vladan Velisavljevic ${ }^{1}$, Eduardo Cano $^{2}$, Vladimir Dyo $^{1}$, Ben Allen $^{3}$ \\ ${ }^{I}$ Department of Computer Science and Technology, University of Bedfordshire \\ Park Square, Luton LU1 3JU, United Kingdom \\ vladan.velisavljevic@beds.ac.uk \\ ${ }^{2}$ European Commission, Joint Research Centre (JRC) \\ Via E. Fermi, 20127 Ispra (VA), Italy \\ ${ }^{3}$ Department of Engineering Science, University of Oxford \\ Parks Road, Oxford OX1 3PJ, United Kingdom
}

\begin{abstract}
Efficiency of transportation of people and goods is playing a vital role in economic growth. A key component for enabling effective planning of transportation networks is the deployment and operation of autonomous monitoring and traffic analysis tools. For that reason, such systems have been developed to register and classify road traffic usage. In this paper, we propose a novel system for road traffic monitoring and classification based on highly energy efficient wireless magnetic sensor networks. We develop novel algorithms for vehicle speed and length estimation and vehicle classification that use multiple magnetic sensors. We also demonstrate that, using such a low-cost system with simplified installation and maintenance compared to current solutions, it is possible to achieve highly accurate estimation and a high rate of positive vehicle classification.
\end{abstract}

Keywords: wireless, magnetic sensors, traffic monitoring, speed and length estimation, vehicle classification

\section{Introduction}

Transportation of people and goods are a fundamental part of the global economy. With a growing world population, demand for an effective transport infrastructure continues to increase at a huge rate, and without careful management, the transportation infrastructure would become highly ineffective. Thus, an intelligent infrastructure that makes use of sensors, communications technology and data analytics is accepted by many to be an enabler for managing an effective transportation system. In terms of the road network, this may entail monitoring road usage for purposes of capacity planning, toll charging and maintenance. These systems typically consist of a power source, sensors, signal conditioning, communications, database and data analytics. There are numerous options for each, influenced by environmental factors, performance requirements and technology availability.

For traffic monitoring, the systems may typically be installed away from a convenient power source. Therefore, they are required to operate autonomously for several years including: classifying, logging and reporting traffic data to a central data centre. Solutions such as those described in (Haoui et al., 2007) and (Cheung et al., 2005) have been designed and investigated. In (Haoui et al., 2007), a single magnetic sensor is used to detect and classify vehicles. This approach has been proposed as a low cost alternative to using classical inductive loops (Ki and Baik, 2006) and it may be deployed quicker and can provide additional detection data not available from inductive loop systems. Signal classification is implemented by means of magnetic signature correlation and comparison to reference signatures. Good results have been shown in terms of correct classification using a single sensor and this achievement sets a benchmark for comparison of the obtained performance reported in this paper. The work reported in (Cheung et al., 2005) presents trials of a commercial product developed using the method from (Haoui et al., 2007). This product uses magnetic sensors and wireless communications technology for reporting the data back to a processing centre. It is shown in (Cheung et al., 2005) that magnetic sensors can provide signatures at least as good as those from inductive loop sensors, but may be more easily deployed. However, the work in (Cheung et al., 2005) neither provides details of the signal processing techniques used nor does it explore the use of multiple sensors.

In addition, some other notable attempts were made to detect and classify road traffic. In (Haijian et al., 2014), classification and regression rate based approach was applied on vehicle magnetic signatures for real-time classification with an accuracy rate of $88.9 \%$ for two vehicle classes. Furthermore, in (Kaewkamnerd et al., 2010), vehicles are classified using a tree algorithm based on signals collected from two anisotropic magneto-resistive sensors achieving an accuracy rate of $81.7 \%$ for 4 classes. Some related 
algorithms were developed for road traffic monitoring using different technologies. For instance, vehicle classification has been implemented in Pretoria, South Africa, using RFID technology (Mbodila et al., 2015), whereas axle counting is used in (Ma et al., 2014). Traffic and infrastructure monitoring was deployed in (Xue et al., 2015), whereas, in (Collotta et al., 2015), traffic detection was developed for use with a traffic light controller.

In contrast to these approaches, we address vehicle classification with multiple classes and our target accuracy rate is higher than the ones achieved by the comparative methods. In collaboration with Clearview Traffic, our project partner, we develop a novel system of wireless magnetic sensor network and advanced signal classification techniques. We demonstrate that high accuracy of vehicle detection and classification can be achieved with only two magnetic sensors, which greatly reduces hardware complexity and costs. In particular, we show how a signal edge detection algorithm successfully used in other areas of signal processing can improve temporal localisation of vehicle drive-by events. By combining magnetic signatures from only two magnetic sensors, the novel proposed method deployed on the newly designed system is capable of providing highly accurate vehicle length and speed estimates. Furthermore, the method is then extended to determine vehicle classification based on the processed magnetic signatures. We evaluate the developed method using a comprehensive database of magnetic signatures recorded in a realistic environment.

The remainder of the paper is organised as follows. In Section 2, we describe the system based on the wireless magnetic sensor network. Then, in Sections 3, we explain the method used for edge localisation in magnetic signatures, which is exploited in Section 4 for vehicle length and speed estimate. In Section 5, we explain the wireless communications protocol. In Section 6, we analyse the obtained performance. Finally, we conclude our findings in Section 7.

\section{System Description}

A generalised system for road traffic monitoring and vehicle classification consists of two parts: a set of sensors and a base station (BS), as illustrated in Figure 1. Each sensor is equipped with magnetometers ( $\mathrm{x}, \mathrm{y}$ and $\mathrm{z}$ axes), a microprocessor with reduced computational power and RF transceiver with antenna. The sensor is connected to the BS via a wireless channel. The BS plays a role of data sink for all the information gathered by the sensors and it deploys an algorithm for vehicle speed estimation and classification.

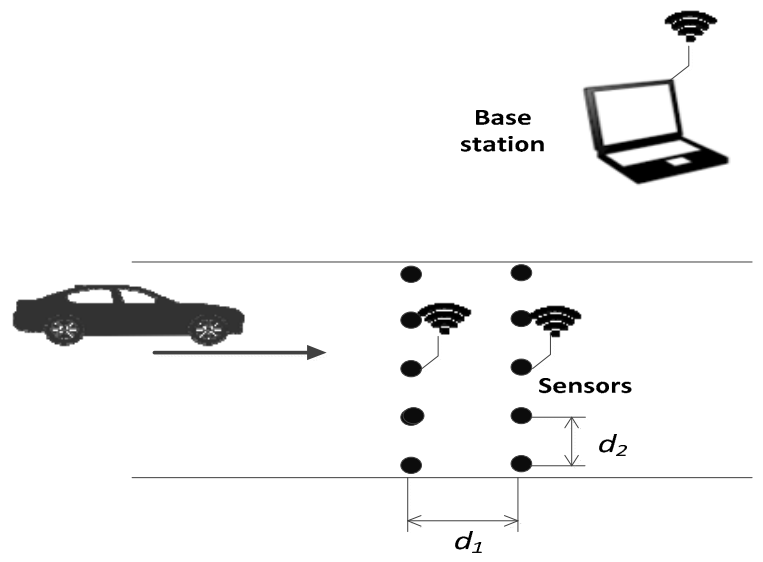

Figure 1. System concept of wireless magnetic sensor network for road traffic monitoring and classification

The number of magnetic sensors is not fixed, but the adopted approach aims at minimising this number to preserve low system costs and simplified installation. For that reason, the target is to have two sensors placed along the road at a fixed distance. The position of this pair of sensors is optimised based on measurements acquired using a comprehensive database provided by Clearview Traffic, as explained in Section 6.

The system processes the magnetic signatures of each vehicle in a two-fold procedure. First, the signatures for each vehicle are captured by the magnetometers in three geometrical axes, i.e. $x, y$ and $z$. These signatures are processed locally by the microprocessor to deploy de-noising and detection of the signature beginning and end time-stamps. The signatures are transferred to the BS via a wireless link along with the detected time-stamps. Then, the BS deploys a processing algorithm to all gathered data 
that estimates the speed and length of the vehicles and classifies them. The results may then be communicated to users. The design of the system has to be such that it fits within the computational resources of the sensors and BS, and within the available energy budget.

The algorithm used for detecting the beginning and end of vehicle magnetic signatures is based on a wavelet multi-scale discontinuity detection method, as explained in the next section. This algorithm is used to extract the two time-stamps corresponding to the beginning and end of the vehicle drive-by event. This is subsequently used to reduce the amount of information and hence data-rate required for communicating and determining vehicle length and speed estimation at the BS. This is explained in detail in the following sections.

\section{Wavelet Multi-Scale Discontinuity Detection}

Singularity or discontinuity detection has been an important challenge in many areas of signal processing, where precise temporal or spatial localisation of events of interest or key signal characteristics plays a fundamental role.

For instance, in image processing, singularities correspond to edges and contours that are important for image understanding and, hence, high popularity of the Canny edge detector (Canny, 1986) in computer vision and image processing. Similarly, in speech processing, discontinuity detection is used for word boundary segmentation (Scharenburg et al., 2010) or for a voice activity cue (Benyassine et al., 1997), which contributes to interference reduction in blind acoustic source separation (Quingju et al., 2014).

In this work, we exploit multi-scale wavelet discontinuity detection to determine the beginning and end of magnetic signatures. The method is used to localise in time the vehicle drive-by events as compared to background noise during no-event time. The original theory was developed in (Mallat and Hwang, 1992; Mallat and Zhong, 1992), whereas the method was then successfully extended to twodimensional signals for image interpolation (Chang et al., 2006).

This method characterizes signal discontinuities across multiple scales of the wavelet transform, where each discontinuity is characterized by amplitude, location and local Lipschitz regularity (Mallat and Zhong, 1992). When the wavelet transform is applied to a signal $f(x)$ with a discontinuity, the resulting wavelet coefficients $W_{s} f(x)$ at scales $s=1,2$,.. contain a footprint that consists of peaks across multiple scales. The locations and amplitudes of the peaks, $\left|W_{s}\right| f(x)$, exhibit coherence across scales so that the locations remain the same, whereas the amplitudes behave as

$\left|W_{s} f(x)\right| \sim K s^{\alpha}$,

where $K$ is a constant, $\alpha$ is the local Lipshitz regularity of the discontinuity and $\sim$ denotes approximate equality. Such a multi-scale coherence of discontinuities is a key feature for providing a robust method to distinguish them from the background noise. For that reason, this method is exploited in our system, as explained next, and is a distinguishing aspect of our system.

\section{Vehicle Length and Speed Estimates}

The method for estimating both vehicle speed and length can be divided into two parts: distributed processing on each sensor microprocessor and centralised processing at the BS. Unlike the BS, the sensor hardware is severely constrained in terms of computational and energy resources and hence the system design criterion is to reduce the energy required for the magnetometers, computation and wireless transmission/reception. First, the wavelet multi-scale discontinuity detection is used to determine the magnetic signature beginning and end on each sensor node, and then this information is used for speed and length estimation conducted centrally at the BS. The speed estimation method has several possible configurations that enable a trade-off between performance and energy efficiency. These are explained in detail below.

\subsection{Detection of Signature Beginning and End}

Vehicle length estimation is based on extracting the location of the signature beginning and end using the detection approach explained in Section 3. This information is communicated to the BS, which performs the final length estimation making use of the estimated speed and sampling frequency $f_{s}$. The method consists of several steps, as follows. 
The magnetometers measure the incident magnetic field in three geometrical axes providing three corresponding components. Since the distribution of the measurements across these three components is sensitive to magnetometer orientation, considering each component separately can affect the estimate and classification performance in practical implementation. For that reason, the magnetic components are processed jointly by computing the magnitudes for each sample as

$m[n]=\sqrt{x[n]^{2}+y[n]^{2}+z[n]^{2}}$,

where $x[n], y[n]$ and $z[n]$ are the three magnetic components at the time instance $n$, and $m[n]$ is the corresponding magnitude. Typical magnetic signature components $x, y$ and $z$ are shown in Figure 2 together with the resultant magnitude.

The magnitude is then transformed using the quadratic spline wavelet transform with the low-pass and high-pass filters $h_{L P}=[1,3,3,1]$ and $h_{H P}=[1,-1]$, respectively, following the method from (Chang et al., 2006). This filter pair is chosen since it provides a good balance between computational efficiency and smoothing, as reported in (Chang et al., 2006), where the same transform was used for image interpolation. The number of wavelet decomposition scales, $J$, in the transform is chosen experimentally so that the mean square error of vehicle length estimate for the available dataset (see Section 6) is minimised. Increasing $J$ leads to better de-noising performance due to iterated low-pass filtering using $h_{H P}$. However, at the same time, such a smoothing operation results in loss of temporal resolution, which deteriorates ability to localise precisely the signature beginning and end. These opposed constraints are best balanced for $J=5$ for use with the database of vehicle magnetic signatures, as explained in Section 6 .

The beginning of the signature is determined as the most prominent positive magnitude discontinuity (i.e. discontinuity with positive amplitude) in the wavelet domain. Similarly, the end of the signature is identified as the most prominent negative magnitude discontinuity. Note that using any of the three separate magnetic components instead of magnitude would cause ambiguity in polarity of the discontinuities since these components are not, unlike the magnitude, constrained to be non-negative. As a consequence, the beginning and end of the signature are always featured by positive and negative discontinuities, respectively. To extract these discontinuities from noisy signatures, they are examined for coherence of the characteristics across wavelet scales given in (1).

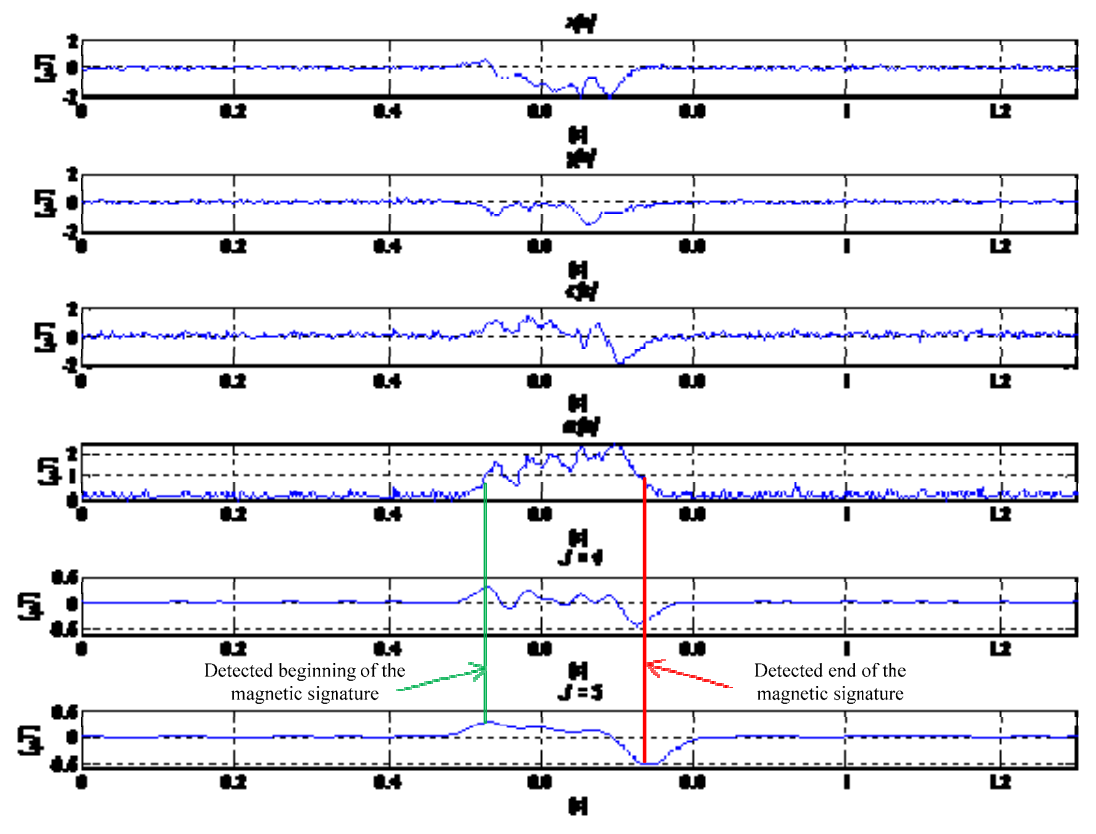

Figure 2. Magnetic field signatures taken at fs $=400 \mathrm{~Hz}$ : three components, $\mathrm{x}, \mathrm{y}, \mathrm{z}$, magnitude $\mathrm{m}$ and wavelet scales at $J=4$ and $J=5$

Since the resultant magnitude exhibits a high level of noise, the first three wavelet scales, $J=1,2,3, .$. , are discarded. Recall that the high-pass outputs of the wavelet transform correspond to derivatives of the iteratively smoothed versions of the original signal. Thus, a positive (or negative) 
discontinuity in the signal will generate a local maximum (or minimum) in the wavelet domain. This property is exploited in the method following (Chang et al., 2006) so that the locations of all local maxima at the scales $J=4$ and 5 are first identified and for the ones that preserve the neighbourhood locations at $J=4$ and $J=5$ (i.e. the local maxima remain in proximity at these two scales) are tested whether they meet the scaling equation (1) for $-1.5 \leq \alpha \leq 1.5$. The local maximum with the minimal location (left-most in the graphs) occurring earliest in time that satisfies these conditions corresponds to the beginning of the signature (denoted in green in Fig. 2). Similarly, the local minimum that satisfies these conditions with the maximal location (right-most in the graphs) occurring latest in time corresponds to the end of the signature (denoted in red in Fig. 2). The indices of the detected beginning and end, $n_{1}$ and $n_{2}$ are communicated to the BS for further processing.

\subsection{Vehicle Speed Estimate}

Speed estimation is based on measuring a time delay, $\Delta t$, between signatures from at least two sensors placed along the road. Knowing the distance, $d$, between these sensors, we can compute the estimated speed, $\hat{v}$, as the ratio $\hat{v}=d / \Delta \hat{t}$, where $\Delta \hat{t}$ is a measured version of $\Delta t$. Note that such an approach inherits a measurement error imposed by using a finite sampling frequency $f_{s}$ because of sampling error in measuring $\Delta t$. Assuming the maximal absolute error in measuring $\Delta t$ equals a half of the sampling interval, $1 / 2 f_{s}$, then the maximal relative error of the estimated speed is given by $\left(1+2 d f_{s} / v\right)^{-1}$. For practical implementation presented in Section 6, this error is not constraining the quality of speed estimate because the reported obtained errors are slightly larger than the theoretical maximal inherited error.

Since the method requires input from at least two sensors, it is implemented centrally at the BS. Note that more accurate speed estimation can be derived by using more than 2 sensors, but since the goal is to minimise the system complexity and costs, we are focusing on speed estimation using only 2 sensors.

Based on the type of input from the sensors and system architecture, we propose three algorithms for measuring the time delay, which allow for trade-off between speed estimation accuracy and computational complexity. In the first Algorithm A, the entire set of signature magnitudes is quantized using $N_{b}$ bits-per-sample (bps) and transmitted from each sensor to the BS. The BS then estimates the time delay between the two signatures as the time instance of the cross-correlation maximum. The two signatures are generated by the same vehicle driving by the two sensors and, thus the shapes of the signatures are expected to be highly correlated. In the experiments, this assumption is verified and can be efficiently exploited. The highest cross-correlation between the two signatures occurs at the time instance equal to the time delay. Fig. 3 shows a typical cross-correlation with a clear peak at the time delay. The number of bps, $N_{b}$, enables scaling of the transmission data-rate versus estimation accuracy and, as explained in Section 6, we have found that the best results are obtained for $N_{b}=5$.

In Algorithm B, the magnitude samples are quantized and transmitted to the BS only for indices between the detected beginning and end of the signatures from each sensor separately. The BS then computes the cross-correlation in the same way as for Algorithm A. The key feature in Algorithm B is to preserve temporal alignment across all used sensors to compute correctly the cross-correlation and this is achieved by attaching time-stamps of each signature to the transmission package in the protocol, as explained in Section 5. Algorithm B reduces the overall transmission data-rate at the cost of a slight performance loss, as analysed below.

Finally, Algorithm C transmits to the BS only the detected beginning and end indices from each sensor. The time delay is estimated as an average difference between the corresponding beginning indices and end indices from the sensors divided by the sampling frequency $f_{s}$. While this algorithm significantly reduces the transmission data-rate, it also results in a lower performance, as analysed in Section 6.

\subsection{Vehicle Length Estimate}

Having the beginning and end indices, $n_{1}{ }^{(i)}$ and $n_{2}{ }^{(i)}$, of signature from the sensor $i=1,2$ transmitted to the BS and the estimated vehicle speed, $\hat{v}$, as explained in Section 4.2, length is calculated as the mean estimate from each sensor, that is,

$\hat{l}=\frac{1}{2} \sum_{i}\left(n_{2}^{(i)}-n_{1}^{(i)}\right) \cdot \frac{\hat{v}}{f_{s}}$. 
Since the length estimate method depends on the speed estimate, there are three algorithms following their definitions in Section 4.2. Note that from (3), the error of speed estimation is propagated to that of length estimation and, thus length estimate accuracy is worse than speed estimate accuracy, as also supported by experimentations and presented in Section 6. Thus, the system design should ensure speed estimation error is minimised.

As a summary, Figure 4 illustrates the block diagrams of the three algorithms.
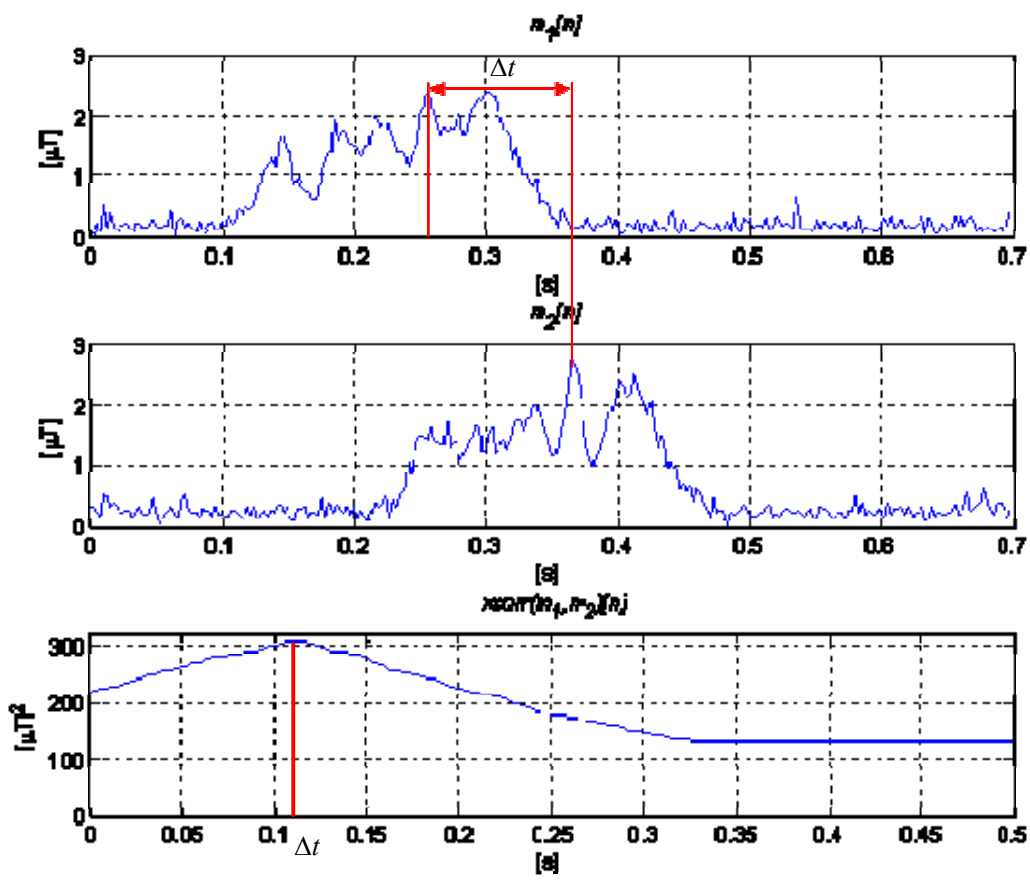

Figure 3. Magnitudes of the magnetic signature from two sensors, (a) and (b), and their cross-correlation with a clear peak (c)

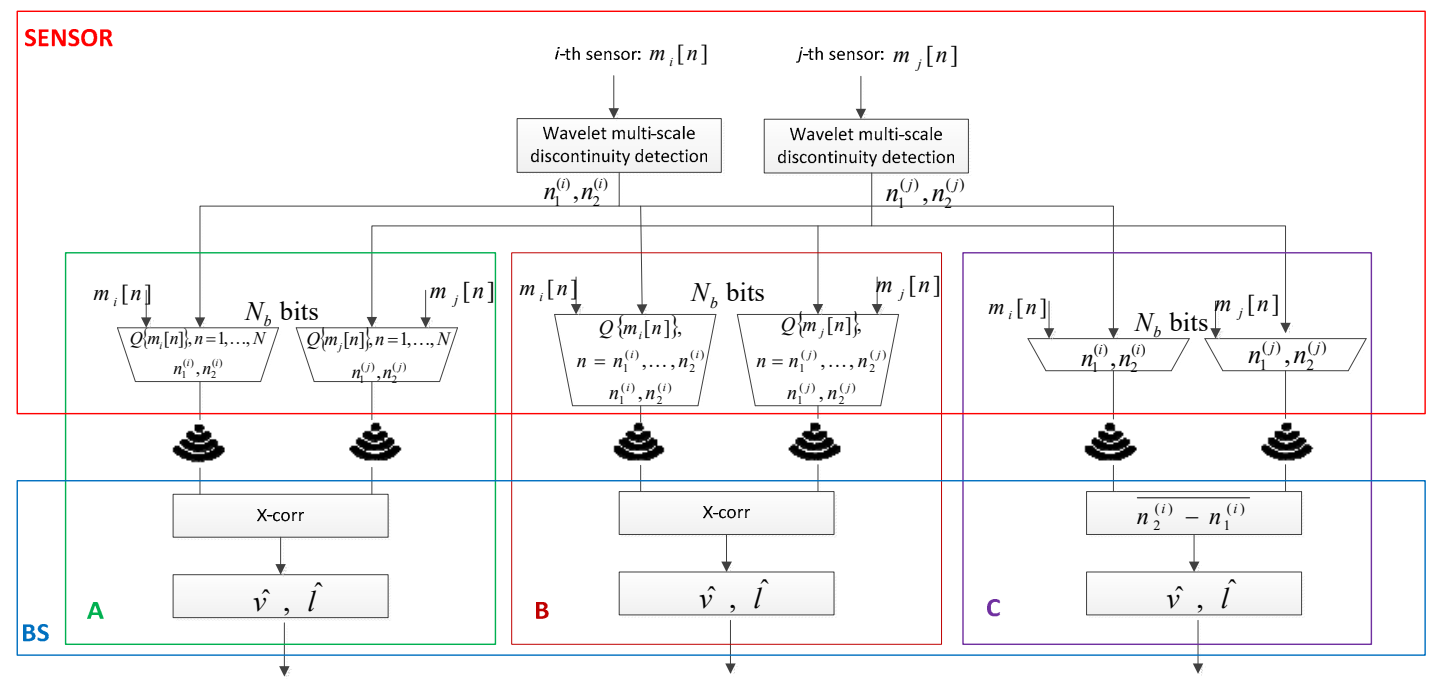

Figure 4. Block diagrams of algorithms A, B and C for vehicle speed and length estimation. Box denoted as SENSOR shows the components of the algorithms implemented on sensor before data transmission, whereas the box BS depicts deployment at BS after data transmission

\section{Wireless Communication Protocol}

The main goal for the wireless communication protocol is to provide data transfer from multiple in-road sensors to a roadside BS in a reliable and timely fashion. Wireless communication is often the 
dominant source of power consumption in wireless sensing applications; hence, it is important to minimize the communication energy costs while meeting the application requirements. While a number of energy efficient MAC protocols have been designed in the past (e.g. (Ye et al., 2006; Buettner et al., 2006)), to the best of our knowledge, none addresses the fact that the communication channel is periodically blocked by passing vehicles. This happens because whenever a vehicle is passing over a sensor node, it significantly degrades the communication channel between the sensor nodes and the BS, causing unnecessary energy wastage on transmission, re-transmission and idle listening.

The proposed protocol combines Time Division Multiple Access (TDMA) and centralised polling to provide robust and energy efficient operation. The key idea of the proposed protocol is to integrate vehicle presence information into a wireless channel access scheme. The diagram illustrating the protocol operation is shown in Fig. 5, whereas the beacon format is presented in Table 1.

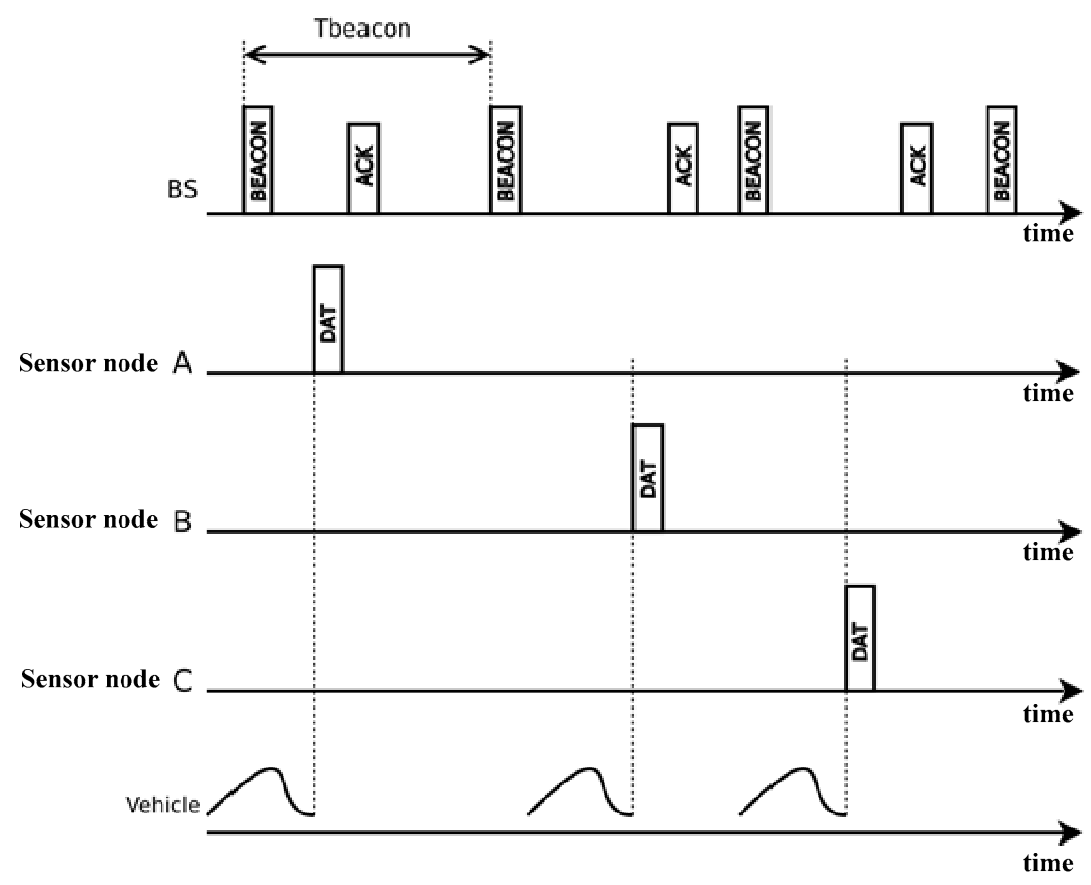

Figure 5. Wireless protocol operation diagram

The protocol operates as follows. The BS transmits a beacon message periodically, to synchronise the nodes and poll the data separately from each sensor. The periodic polling allows the protocol to cope with high data-rate scenarios, e.g. due to higher sampling frequency, number of samples or when a large number of sensors is deployed, while eliminating the possibility of packet collisions.

Table 1. Beacon format

\begin{tabular}{|l|l|}
\hline Field & Length (bits) \\
\hline BS ID & 32 \\
\hline Beacon interval & 32 \\
\hline Sensor-to-poll & 32 \\
\hline Time-stamp & 32 \\
\hline
\end{tabular}

\subsection{Initialisation}

Each sensor node enters into standby mode upon start-up, where it searches for beacons by turning on its receiver for $T_{\text {beacon }}$ seconds every $T_{\text {beacon }} \times N_{\text {skip }}$ seconds. Each beacon contains the duration of a beacon interval $T_{\text {beacon }}$ and a time-stamp indicating the current time at a BS. Once the node receives a beacon, it computes the clock offset between the local clock and BS, schedules its wake-up for the next beacon and switches into data transfer mode, described below. $N_{\text {skip }}$ is a fixed parameter based on the available energy budget and the maximum latency for a BS discovery. Lower values of $N_{\text {skip }}$ allow the sensor node to discover the BS quicker, at the expense of higher energy consumption. 


\subsection{Data Transfer}

Once the sensor node receives a beacon, it compares the beacon's Sensor-to-poll field with its own ID, and initiates a data transfer if it matches, or sleeps until the next beacon. Reliable data transfer is done using a simple ARQ protocol, where each data packet is acknowledged by a BS. No acknowledgement indicates either a lost data packet or lost acknowledgement, and since both events are indistinguishable to the sender, it retransmits any unacknowledged packet again (up to $N_{\text {retrans }}$ attempts after which the transmission is considered failed and the packet is discarded). Each packet contains a sequence number incremented upon each successful transmission. The sequence number is used by the BS to detect gaps in the stream of packets.

To take into account the presence of a vehicle, the protocol checks the magnetic sensor reading before a transmission begins. Data transfer starts immediately if the measured intensity of the magnetic field is less than $M_{\text {thresh }}$. Otherwise, the node assumes there is a vehicle in the proximity, turns off the transceiver and waits until the magnetic field intensity drops below $M_{t h r e s h}$. The vehicle presence can also affect the beacon transmission itself, and while this cannot be avoided in some cases, it is mitigated by using a higher TX power from the BS, which may be possible due to its higher energy budget. If a sensor node does not hear from the BS for more than $N_{\text {lost }}$ seconds (for example, due to a vehicle remaining in the proximity), it switches into an energy-saving standby mode, waking-up periodically to detect a beacon from the BS as described in Section 5.1. As soon as the beacon is detected, the node switches back into the data transfer mode.

\subsection{Data Processing}

The set of magnetic sensor samples that form a magnetic signature from a vehicle are organised into data records of the format shown in Table 2.

Table 2. Data record format

\begin{tabular}{|l|l|}
\hline Field & Length (bits) \\
\hline Local time-stamp & 32 \\
\hline Offset & 16 \\
\hline Length & 16 \\
\hline Data & $\ldots$ \\
\hline Padding & $0, \ldots, 7$ \\
\hline
\end{tabular}

The offset field allows splitting long data records across several packets. The overall packet contains several records and has the format detailed in Table 3.

Table 3. Packet format

\begin{tabular}{|l|l|}
\hline Field & Length (bits) \\
\hline Source address & 32 \\
\hline Destination address & 32 \\
\hline Sequence number & 8 \\
\hline Local time-stamp & 32 \\
\hline Flags & 8 \\
\hline Data length & 16 \\
\hline Checksum & 16 \\
\hline Record $\# 1$ & \\
\hline Record $\# 2$ & \\
\hline$\ldots$ & \\
\hline Record $\# N$ & \\
\hline
\end{tabular}

The sequence number is incremented upon each successful transmission and is used by the BS to detect missing packets.

The BS receives the packets and performs the actions listed in Table 4. 
Table 4. BS actions

\begin{tabular}{|l|l|}
\hline Step & Action \\
\hline 1. & Send an acknowledgement immediately. \\
\hline 2. & $\begin{array}{l}\text { Use packet sequence numbers to discard repetitive data. Duplicated packets with the same sequence number occur when } \\
\text { the acknowledgement is lost and the sensor node retransmits the packet. }\end{array}$ \\
\hline 3. & $\begin{array}{l}\text { Use packet sequence number to detect missing data. In case a missing packet is detected, it discards relevant fragments } \\
\text { from the same node and the relevant samples from other nodes. }\end{array}$ \\
\hline 4. & Convert the event local time-stamps within the packet to a global time reference. \\
\hline 5. & Re-assembles long records that are split among several packets. \\
\hline 6. & Re-assembles complete events (samples from several nodes that belong to the same event) based on these time-stamps. \\
\hline 7. & Passes on the complete event to a vehicle classification module. \\
\hline
\end{tabular}

\section{Performance Analysis}

Experimentation and performance evaluation were carried out in collaboration with Clearview Traffic. A set of 32 magnetometer sensor nodes was deployed on a public road B-4100 in the area of Bicester, Oxfordshire, UK, to collect vehicle magnetic signatures, as illustrated in Fig. 6(a). These sensors collected samples from 930 vehicles classified into 8 types according to the classification given in Table 5. Each signature is annotated with the type of vehicle identified visually from the associated video record and with ground-truth speed and length obtained from a magnetic loop device installed for benchmarking in parallel to the sensor network. Next, we show how the best pair of sensors is selected and we demonstrate accuracy rates of the speed and length estimate and the vehicle classification.

Table 5. Vehicle classification and number of vehicles in each category according to the video data

\begin{tabular}{|l|l|l|}
\hline Class & Description & Actual number of vehicles \\
\hline$(1)$ & Motorbikes & 5 \\
\hline$(2)$ & Car or light van & 713 \\
\hline$(3)$ & Car or light van plus trailer & 10 \\
\hline$(4)$ & Heavy van or minibus & 111 \\
\hline$(5)$ & Rigid (LGV) & 31 \\
\hline$(6)$ & Rigid (MGV) & 25 \\
\hline$(7)$ & Rigid plus trailer & 20 \\
\hline$(8)$ & Bus or coach & 15 \\
\hline \hline Total & 930 \\
\hline
\end{tabular}

\subsection{Selection of the best pair of sensors}

The 32 sensors were installed in two linear arrays with 16 sensors in each row separated by a distance of $d=d_{1}=3 \mathrm{~m}$. Since the goal is to keep only 2 sensors, we select the best pair of sensors aligned with the road direction based on the criterion of highest average Signal-to-Noise-Ratio (SNR) of the magnetic signatures for the vehicles in the collected database. The SNR for different sensors depends mostly on the sensor location across the road - if vehicles are driven close to the sensor, the SNR will be higher and the signature will be clearer. Thus, we compute the average SNR (the mean between the SNRs obtained from the two sensors for each pair) for all 16 pairs of sensors and select the strongest SNR. Fig. 6(b) shows the SNRs for these 16 combinations with the pair (6-22) being the best one. Hence, we process further only the signatures from these two sensors.

\subsection{Speed and length estimation accuracy}

The acquired database of vehicle magnetic signatures has been captured at the sampling frequency $f_{s}=400 \mathrm{~Hz}$. For the purpose of a more detailed performance analysis, we will analyse speed and length estimation accuracy for four different sampling frequencies, $f_{S} \in\{400,200,133,100\} \mathrm{Hz}$, obtained by subsampling the original signatures.

The signatures are quantized using $2 \leq N_{b} \leq 6$ bps so that the range between zero and maximal magnitude in the signature is divided in $2^{N_{b}}$ uniform quantization intervals. Note that such an adaptive quantization approach requires transmitting the maximal magnitude to the receiver for successful reconstruction of the quantized samples. 


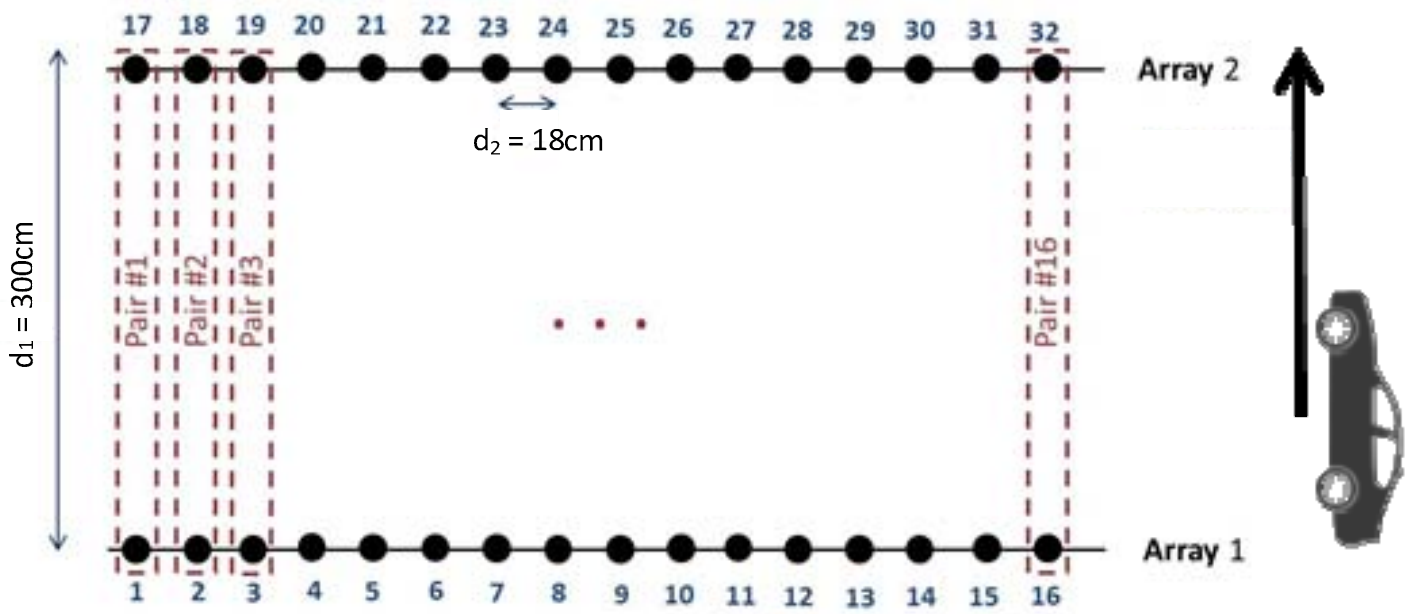

(a)

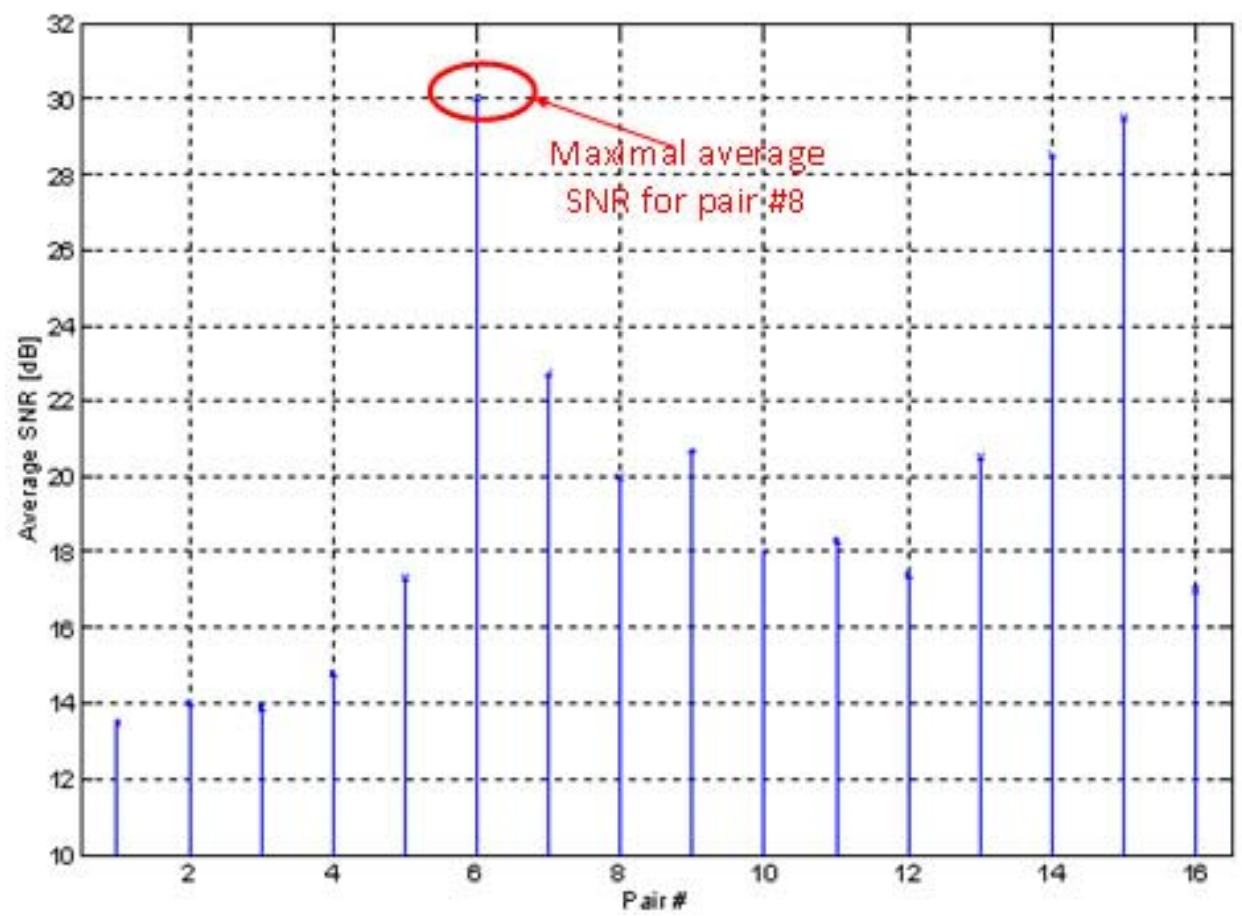

(b)

Figure 6. (a) Layout of 32 magnetometer sensors deployed on a public road.

(b) Average SNR for each of 16 sensor pairs obtained as the mean for two SNRs from each sensor in the pair. The sensor pair \#6 exhibits the highest SNR and is selected for further processing

Accuracy of the speed and length estimation is now determined for the three algorithms A, B and $\mathrm{C}$, as described in Section 4. The obtained performance is compared in terms of two accuracy metrics: Mean-Relative-Error (MRE) and Mean-Absolute-Error (MAE). For speed estimation, the MRE is defined as $M R E_{v}=\overline{|\hat{v}-v| / v}$, whereas the MAE is defined as $M A E_{v}=\overline{|\hat{v}-v|}$, where the ground-truth values are obtained using the annotation in the database acquired using the magnetic loop system. The metrics for length estimate accuracy, $M R E_{l}$ and $M A E_{l}$ are obtained in a similar way to the ones for speed estimation. All the metric values are shown in Table 6, where $M R E$ is expressed in terms of \%, $M A E_{v}$ as $\mathrm{km} / \mathrm{h}$ and $M A E_{l}$ as $\mathrm{cm}$. Note that quantization is not used in Algorithm $\mathrm{C}$ and, thus, $N_{b}$ does not affect the performance in the right-hand side columns. Note also that Algorithm A has the best performance for speed estimate and remains stable for a range of $N_{b}$ and $f_{s}$. For length estimation, Algorithms A and B have comparable performance. Algorithm $\mathrm{C}$ is inferior to the other two in all cases, which is expected since it operates at a significantly reduced transmission data-rate, but also has lower resulting power requirements, as analysed below. 
Figure 7(a) shows the speed estimation performance of Algorithm A in the case of $f_{s}=200 \mathrm{~Hz}$ and $N_{b}=5$ bps. The graph differentiates the performance for each class of vehicles, as listed in Table 5 . Similarly, Figure 7(b) illustrates the length estimate performance for the same case. Note that separation of classes using length is quite clearly visible in Figure 7(b), which is exploited for vehicle classification, as explained in Section 6.3.

Table 6. Speed and length accuracy evaluation. The metrics $M R E_{v}$ (in terms of \%), $M A E_{v}(\mathrm{~km} / \mathrm{h}), M R E_{l}(\%)$ and $M A E_{l}(\mathrm{~cm})$ are shown for several values of the sampling frequency $f_{s}$ and number of quantization bps $N_{b}$. The values for MRE are expressed in terms of $\%$, whereas the ones for MAE are given in $\mathrm{km} / \mathrm{h}$ and $\mathrm{cm}$ for speed and length, respectively

\begin{tabular}{|c|c|c|c|c|c|c|c|c|c|c|c|c|c|}
\hline \multirow{2}{*}{$\begin{array}{l}f_{s} \\
(\mathrm{~Hz})\end{array}$} & \multirow{2}{*}{$\begin{array}{l}N_{b} \\
\text { (bps) }\end{array}$} & \multicolumn{4}{|c|}{ Algorithm A } & \multicolumn{4}{|c|}{ Algorithm B } & \multicolumn{4}{|c|}{ Algorithm C } \\
\hline & & $M R E_{v}$ & $M A E_{v}$ & $M R E_{l}$ & $M A E_{l}$ & $M R E_{v}$ & $M A E_{v}$ & $M R E_{l}$ & $M A E_{l}$ & $M R E_{v}$ & $M A E_{v}$ & $M R E_{l}$ & $M A E_{l}$ \\
\hline \multirow[t]{5}{*}{100} & 2 & 6.80 & 6.50 & 28.62 & 137.02 & 13.32 & 12.31 & 32.11 & 154.84 & \multirow[t]{5}{*}{14.91} & \multirow[t]{5}{*}{13.88} & \multirow[t]{5}{*}{31.47} & \multirow[t]{5}{*}{155.67} \\
\hline & 3 & 6.52 & 6.24 & 28.50 & 135.69 & 11.78 & 10.93 & 29.78 & 144.04 & & & & \\
\hline & 4 & 6.55 & 6.27 & 28.50 & 135.49 & 11.56 & 10.74 & 29.53 & 143.07 & & & & \\
\hline & 5 & 6.54 & 6.25 & 28.55 & 135.68 & 11.80 & 10.96 & 29.62 & 143.73 & & & & \\
\hline & 6 & 6.51 & 6.23 & 28.62 & 136.08 & 11.87 & 11.04 & 29.70 & 144.11 & & & & \\
\hline \multirow[t]{5}{*}{133} & 2 & 6.69 & 6.34 & 21.79 & 105.06 & 10.16 & 9.39 & 20.74 & 106.04 & \multirow[t]{5}{*}{11.87} & \multirow[t]{5}{*}{10.95} & \multirow[t]{5}{*}{20.52} & \multirow[t]{5}{*}{1110.54} \\
\hline & 3 & 6.29 & 5.96 & 21.66 & 104.33 & 9.48 & 8.76 & 20.04 & 103.19 & & & & \\
\hline & 4 & 6.06 & 5.78 & 21.38 & 103.22 & 9.59 & 8.83 & 20.08 & 103.42 & & & & \\
\hline & 5 & 6.09 & 5.82 & 21.39 & 103.24 & 9.60 & 8.82 & 19.82 & 102.63 & & & & \\
\hline & 6 & 6.03 & 5.75 & 21.33 & 102.84 & 9.56 & 8.79 & 19.97 & 102.98 & & & & \\
\hline \multirow[t]{5}{*}{200} & 2 & 6.74 & 6.40 & 18.00 & 992.84 & 7.89 & 7.36 & 18.79 & 96.42 & \multirow[t]{5}{*}{8.90} & \multirow[t]{5}{*}{8.41} & \multirow[t]{5}{*}{19.13} & \multirow[t]{5}{*}{98.56} \\
\hline & 3 & 6.32 & 6.00 & 17.81 & 91.71 & 7.58 & 7.02 & 18.48 & 94.63 & & & & \\
\hline & 4 & 6.06 & 5.80 & 17.54 & 90.43 & 7.36 & 6.87 & 18.23 & 93.58 & & & & \\
\hline & 5 & 6.05 & 5.78 & 17.56 & 90.46 & 7.19 & 6.71 & 18.31 & 93.94 & & & & \\
\hline & 6 & 6.07 & 5.80 & 17.58 & 90.51 & 7.20 & 6.73 & 18.33 & 94.05 & & & & \\
\hline \multirow[t]{5}{*}{400} & 2 & 6.50 & 6.15 & 14.41 & $\begin{array}{l}76.04 \\
\end{array}$ & 6.57 & 6.22 & \begin{tabular}{c|c|}
14.38 \\
\end{tabular} & $\begin{array}{l}75.67 \\
\end{array}$ & \multirow[t]{5}{*}{7.85} & \multirow[t]{5}{*}{7.38} & \multirow[t]{5}{*}{15.05} & \multirow[t]{5}{*}{82.81} \\
\hline & 3 & 6.13 & 5.82 & 14.12 & 74.53 & 6.28 & 5.95 & 14.14 & 74.68 & & & & \\
\hline & 4 & 5.87 & 5.61 & 13.90 & 73.46 & 6.05 & 5.78 & 13.96 & 73.90 & & & & \\
\hline & 5 & 5.87 & 5.61 & 13.87 & 73.34 & 6.07 & 5.79 & 13.91 & 73.65 & & & & \\
\hline & 6 & 5.86 & 5.60 & 13.87 & 73.30 & 6.05 & 5.77 & 13.92 & 73.69 & & & & \\
\hline
\end{tabular}

Table 7. Comparison of transmission data-rates and computational loads on sensor microprocessor for Algorithms A, B and C. Both metrics are expressed per signature and sensor. Number of mathematical operations is approximatively independent of $N_{b}$

\begin{tabular}{|c|c|c|c|c|c|c|c|}
\hline \multirow[t]{2}{*}{$f_{s}(\mathrm{~Hz})$} & \multirow{2}{*}{$\begin{array}{l}N_{b} \\
\text { (bps) }\end{array}$} & \multicolumn{2}{|l|}{ Algorithm A } & \multicolumn{2}{|l|}{ Algorithm B } & \multicolumn{2}{|l|}{ Algorithm C } \\
\hline & & $\begin{array}{l}\text { bits per sign- } \\
\text { sensor }\end{array}$ & $\begin{array}{l}\text { \# comp per } \\
\text { sign-sensor }\end{array}$ & $\begin{array}{l}\text { bits per sign- } \\
\text { sensor }\end{array}$ & $\begin{array}{l}\text { \# comp per } \\
\text { sign-sensor }\end{array}$ & $\begin{array}{l}\text { bits per sign- } \\
\text { sensor }\end{array}$ & $\begin{array}{l}\text { \# comp per } \\
\text { sign-sensor }\end{array}$ \\
\hline \multirow[t]{5}{*}{100} & 2 & 482 & \multirow[t]{5}{*}{$\sim 6757$} & 86 & \multirow[t]{5}{*}{$\sim 6757$} & \multirow[t]{5}{*}{32} & \multirow[t]{5}{*}{$\sim 6524$} \\
\hline & 3 & 716 & & 113 & & & \\
\hline & 4 & 949 & & 140 & & & \\
\hline & 5 & 1182 & & 168 & & & \\
\hline & 6 & 1415 & & 195 & & & \\
\hline \multirow[t]{5}{*}{133} & 2 & 638 & \multirow{5}{*}{ 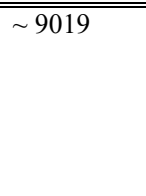 } & 99 & \multirow[t]{5}{*}{$\begin{array}{c}\sim 9019 \\
\end{array}$} & \multirow[t]{5}{*}{32} & \multirow[t]{5}{*}{$\sim 88708$} \\
\hline & 3 & 949 & & 132 & & & \\
\hline & 4 & 1259 & & 166 & & & \\
\hline & 5 & 1570 & & 199 & & & \\
\hline & 6 & 1881 & & 233 & & & \\
\hline \multirow[t]{5}{*}{200} & 2 & 948 & \multirow[t]{5}{*}{$\sim 13514$} & 127 & \multirow[t]{5}{*}{$\sim 13514$} & \multirow[t]{5}{*}{32} & \multirow[t]{5}{*}{${ }^{\prime \sim 13048}$} \\
\hline & 3 & 1414 & & 174 & & & \\
\hline & 4 & 1881 & & 222 & & & \\
\hline & 5 & 2347 & & 269 & & & \\
\hline & 6 & 2813 & & 317 & & & \\
\hline \multirow[t]{5}{*}{400} & 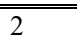 & 1880 & \multirow[t]{5}{*}{$=27028$} & 218 & \multirow[t]{5}{*}{$=27028$} & \multirow[t]{5}{*}{32} & \multirow[t]{5}{*}{ P 26096} \\
\hline & 3 & 2812 & & 310 & & & \\
\hline & 4 & 3744 & & 403 & & & \\
\hline & 5 & 4676 & & 496 & & & \\
\hline & 6 & 5608 & & 589 & & & \\
\hline
\end{tabular}




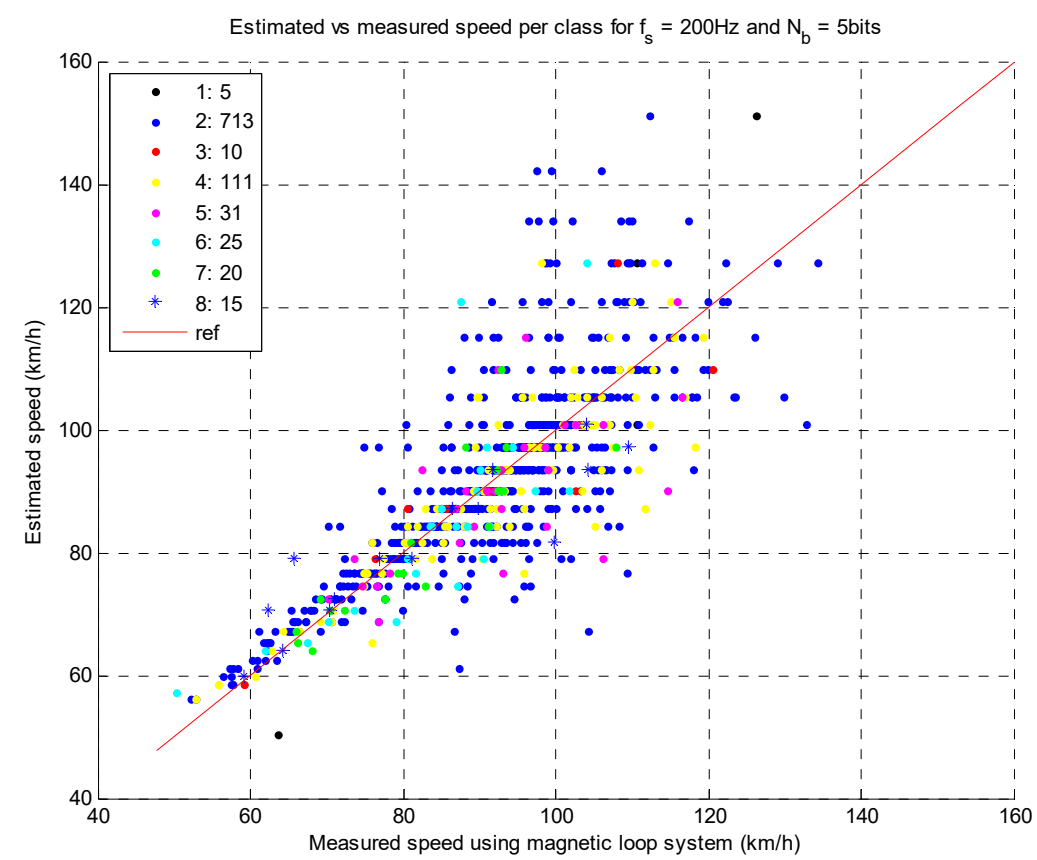

(a)

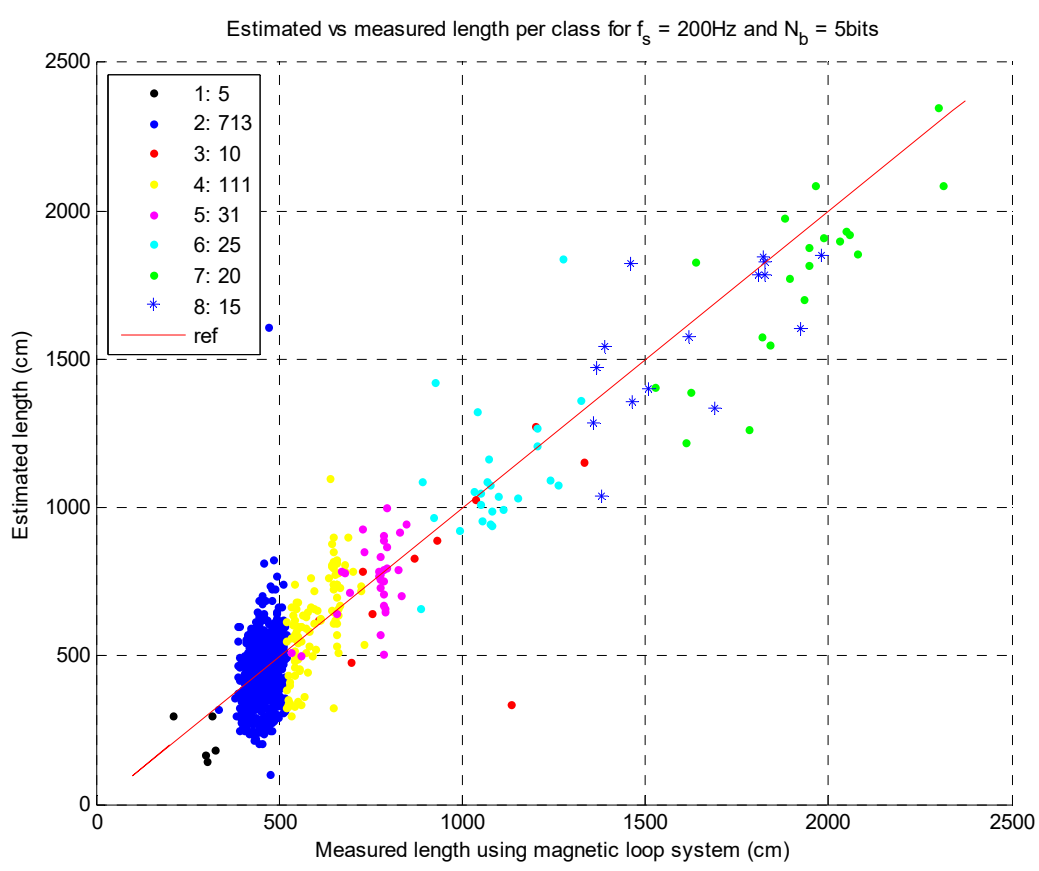

(b)

Figure 7. (a) Speed estimate performance of Algorithm A for each vehicle class for $\mathrm{f}_{\mathrm{s}}=200 \mathrm{~Hz}$ and $\mathrm{N}_{\mathrm{b}}=5 \mathrm{bps}$. The estimated speed takes discrete values because of a finite resolution of cross-correlation. (b) Length estimate performance in the same case

Algorithms $\mathrm{A}, \mathrm{B}$ and $\mathrm{C}$ differ significantly in terms of required transmission data-rate and computational load on the sensor microprocessors. Table 7 shows an overview of these two metrics for all three algorithms. It can be observed from Tables 6 and 7 that performance may be traded-off for resource usage (transmission data-rate and computational load) at very fine granularity by choosing different algorithms and parameters $f_{s}$ and $N_{b}$. 


\subsection{Vehicle classification accuracy}

Our vehicle classification method presented in this section is based solely on estimated vehicle lengths using thresholding as the classification criterion due to the need to preserve low computational complexity. We leave investigation of the application of more sophisticated classification methods for the future work.

Given a respectable database size, list of classes in Table 5 and vehicle lengths shown in Figure 7 , we consider classification without using Class (3) because the vehicles from this class are scattered in both real and estimated length, thus mixing with other classes. For similar reasons, we consider Classes (7) and (8) together as a joint class of lengthy vehicles and Classes (1) and (2) as motorbike-car joint class due to a small number of recorded motorbikes. Therefore, 5 separate classes are used for the evaluation purposes delimited by 4 thresholds. The histogram of vehicle lengths belonging to the 5 classes is graphically shown in Figure 8.

Classification performance is evaluated by comparing the true-positive-rate (TPR) for various cases. The TPR is obtained as a ratio between correct vehicle classification occurrences and total number of vehicles and, hence, it is upper-bounded by $100 \%$. The process of classification into 5 classes in each case is optimised so that the 4 corresponding length delimiter thresholds are chosen based on estimated lengths such that the TPR is maximised. In parallel, classification based on real lengths is deployed to produce a benchmark TPR. Table 8 shows the TPRs for all three algorithms and a range of $f_{s}$ and $N_{b}$ and it compares them to the benchmark TPR. Note that the achieved classification results are close to the benchmark TPR even though only a simple comparison of estimated length is used as the classification core engine.

In addition, we compare this performance to the achievements reported in (Cheung et al., 2005). Unfortunately, not enough technical details of the corresponding method are provided in the relevant publication and we have not been able to apply the method to our database. However, the reported quantitative performance metric in (Cheung et al., 2005) shows that for the database of 37 vehicles only $65 \%$ of vehicles (24) were classified correctly using 5 vehicle classes and that rate raised to $83 \%(31$ vehicles) when using 3 classes. While these rates may be acceptable in some applications, our proposed methods exhibit a higher classification performance that remains robust and stable for a much larger vehicle database.

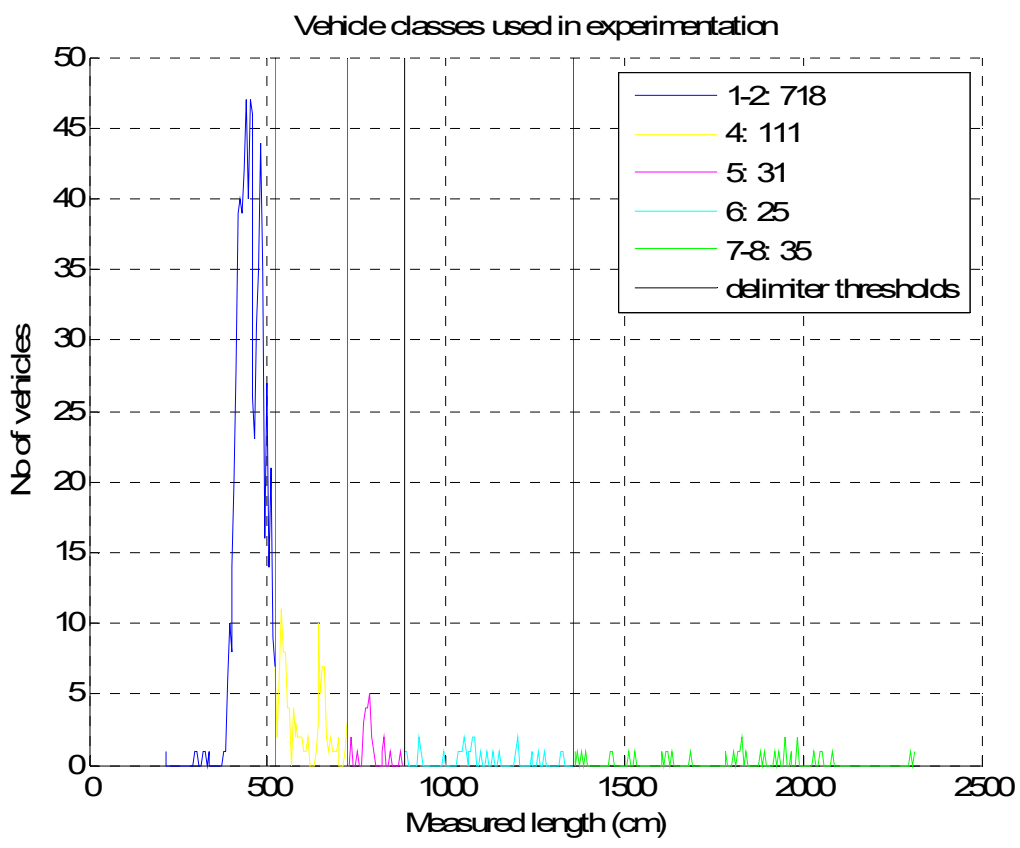

Figure 8. Optimal delimiter thresholds for vehicles classification into 5 classes based on measured lengths. The optimal length delimiter thresholds are as follows: $\{520,726,886,1358\} \mathrm{cm}$ 
Table 8. TPRs for a range of $f_{s}$ and $N_{b}$ and for all three algorithms. The benchmark TPR obtained using real length is shown for comparison

\begin{tabular}{|c|c|c|c|c|}
\hline \multirow[t]{2}{*}{$f_{s}(\mathrm{~Hz})$} & \multirow[t]{2}{*}{$N_{b}$ (bps) } & \multicolumn{3}{|c|}{ Benchmark TPR $=99.24 \%$} \\
\hline & & Algorithm A & Algorithm B & Algorithm C \\
\hline \multirow[t]{5}{*}{100} & 2 & $82.50 \%$ & $80.43 \%$ & \multirow[t]{5}{*}{$81.96 \%$} \\
\hline & 3 & $82.72 \%$ & $82.07 \%$ & \\
\hline & 4 & $82.61 \%$ & $82.39 \%$ & \\
\hline & 5 & $82.83 \%$ & $83.15 \%$ & \\
\hline & 6 & $82.83 \%$ & $82.93 \%$ & \\
\hline \multirow[t]{5}{*}{133} & 2 & $84.67 \%$ & $84.46 \%$ & \multirow[t]{5}{*}{$84.02 \%$} \\
\hline & 3 & $85.33 \%$ & $85.11 \%$ & \\
\hline & 4 & $85.22 \%$ & $85.00 \%$ & \\
\hline & 5 & $85.43 \%$ & $85.11 \%$ & \\
\hline & 6 & $85.43 \%$ & $84.89 \%$ & \\
\hline \multirow[t]{5}{*}{200} & 2 & $87.28 \%$ & $87.07 \%$ & \multirow[t]{5}{*}{$85.54 \%$} \\
\hline & 3 & $86.96 \%$ & $86.74 \%$ & \\
\hline & 4 & $87.07 \%$ & $86.85 \%$ & \\
\hline & 5 & $86.96 \%$ & $86.96 \%$ & \\
\hline & 6 & $86.96 \%$ & $86.96 \%$ & \\
\hline \multirow[t]{5}{*}{400} & 2 & $87.93 \%$ & $88.15 \%$ & \multirow[t]{5}{*}{$86.63 \%$} \\
\hline & 3 & $87.83 \%$ & $88.15 \%$ & \\
\hline & 4 & $88.37 \%$ & $88.26 \%$ & \\
\hline & 5 & $88.04 \%$ & $88.37 \%$ & \\
\hline & 6 & $87.93 \%$ & $88.37 \%$ & \\
\hline
\end{tabular}

\section{Conclusions}

Automatic monitoring and analysis of road traffic is essential for effective planning of transportation networks. While classical systems based on magnetic loop technology can provide reliable traffic data, they are cost ineffective and complicated to install and maintain. For that reason, we have proposed a novel system that uses a pair of wireless magnetic sensors to measure the magnetic signatures generated by passing vehicles. Furthermore, we develop novel algorithms for vehicle speed and length estimation and classification using these signatures. We demonstrate that it is possible to achieve reliable estimation and classification using a reduced-cost system and energy-efficient processing, which is supported by extensive experimentation using a comprehensive database gathered in a realistic scenario. Our system was shown to achieve classification accuracy rate of $88.37 \%$ with 5 vehicle classes when processing a large realistic dataset, which outperforms relevant competitive methods, such as (Cheung et al., 2005) (65\%) and (Kaewkamnerd et al., 2010) (81.7\%). In addition, our system exhibits a similar performance as that of the method proposed in (Haijian et al., 2014) $(88.9 \%)$, which is constrained to work with only 2 vehicle classes. Finally, unlike the competitive methods, our system design enables efficient trading of estimation accuracy for computational complexity and transmission data-rate.

While the achieved accuracy rates are competitive, we envisage that they can be pushed further up by deploying more sophisticated classification algorithms in cases when power constraints are less severe. For instance, classification based on support-vector-machine has a superior performance and can work efficiently with multiple classes. Furthermore, relaxing the system complexity constraint would allow for improved performance by installing more magnetic sensors, where optimisation of the sensor layout might play an important role on the vehicle detection and classification. We leave these points for the future consideration.

\section{Acknowledgements}

This work was carried out in collaboration with Clearview Traffic and was funded by the UK's innovation agency, Innovate UK, Project No. 131603. 


\section{References}

1. Benyassine, A., Shlomot, E., Su, H.-Y., Massaloux, D., Lamblin, C., Petit, J.-P. (1997) ITU-T Recommendation G.729 optimized for V.70 digital simultaneous voice and data applications, IEEE Communications Magazine, vol. 35, no. 9, pp. 64-73.

2. Buettner, M., Yee, G.V., Anderson, E., Han, R. (2006) X-MAC: A short preamble MAC protocol for duty-cycled wireless sensor networks, In: Proceedings of the ACM Conf. on Embedded Networked Sensor Systems (SenSys), Boulder, CO, USA.

3. Canny, J.F. (1986) A computational approach to edge detection, IEEE Transactions on Patern Analysis and Machine Intelligence, vol. 8, no. 6, pp. 679-698.

4. Chang, S.G., Cvetkovic, Z., Vetterli, M. (2006) Locally adaptive wavelet-based image interpolation, IEEE Transactions on Image Processing, vol. 15, no. 6, pp. 1471-1485.

5. Cheung, S.Y., Coleri, S., Dunbar, B., Ganesh, S., Tan, C.-W., Varaiya, P. (2005) Traffic Measurement and Vehicle Classification with Single Magnetic Sensor, Journal of the Transportation Research Board (Transportation Research Record), pp. 173-181.

6. Collotta, M., Lo Bello, L., G. Pau, G. (2015) A novel approach for dynamic traffic lights management based on wireless sensor networks and multiple fuzzy logic controllers, Expert Systems with Applications, vol. 42, no. 13, pp. 5403-5415.

7. Haijian, L., Honghui, D., Limin, J., Moyu, R. (2014) Vehicle classification with single multifunctional magnetic sensor and optimal MNS-based CART, Measurement: Journal of the International Measurement Confederation, vol. 55, pp. 142-152.

8. Haoui, A., Kavaler, R., Varaiya, P. (2007) Wireless magnetic sensors for traffic surveillance, Elsevier Transportation Research Part $C$.

9. Kaewkamnerd, S., Chinrungrueng, J., Pongthornseri, R., Dumnin, S. (2010) Vehicle classification based on magnetic sensor signal, In: Proceedings of the IEEE Int. Conf. on Information and Automation (ICIA).

10. Ki, Y.K., Baik, D.K. (2006) Vehicle-classification algorithm for single-loop detectors using neural networks, IEEE Transactions on Vehicular Technologies, vol. 55, no. 6.

11. Ma, W., Xing, D., McKee, A., Bajwa, R., Flores, C., Fuller, B., and Varaiya, P. (2014) A wireless accelerometer-based automatic vehicle classification prototype system, IEEE Trans. on Intelligent Transportation Systems, vol. 15, no. 1.

12. Mallat, S., Hwang, W.L. (1992) Singularity detection and processing with wavelets, IEEE Transactions on Information Theory, vol. 38, no. 2, pp. 617-643.

13. Mallat, S., Zhong, S. (1992) Characterization of signals from multiscale edges, IEEE Transactions on Pattern Analysis and Machine Intelligence, vol. 14, no. 7, pp. 2207-2232.

14. Mbodila, M, Obeten, E., Bassey, I. (2015) Implementation of novel vehicles' traffic monitoring using wireless sensor network in South Africa, In: Proceedings of the IEEE Int. Conf. on Communication Software and Networks (ICCSN).

15. Qingju, L., Aubrey, A.J., Wenwu, W. (2014) Interference reduction in reverberant speech separation with visual voice activity detection, IEEE Transactions on Multimedia, vol. 16, no. 6, pp. 1610-1623.

16. Scharenborg, O., Wan, V., Ernestus, M. (2010) Unsupervised speech segmentation: an analysis of the hypothesized phone boundaries, The Journal of the Acoustical Society of America, vol. 127, no. 2, pp. 1084-1095.

17. Xue, W., Wang, L., Wang, D. (2015) A prototype integrated monitoring system for pavement and traffic based on an embedded sensing network, IEEE Trans. on Intelligent Transportation Systems, vol. 16, no. 3, pp. 1380-1390.

18. Ye, W., Silva, F., Heidemann, J. (2006) Ultra-low duty cycle MAC with scheduled channel polling, In: Proceedings of the ACM Conf. on Embedded Networked Sensor Systems (SenSys), Boulder, CO, USA. 